\title{
Estimate of Penetration Depth of Moist Soils For Mosul City at 6 GHz C-band Microwave Frequency Range
}

\author{
Manaf Ezzldien Al-Sabbagh \\ Electrical Engineering Department \\ College of Engineering \\ University of Mosul
}

\begin{abstract}
Results of an experimental and theoretical programs were presented to determine the impact of soil moisture content and the soil composition on microwave radiometric emission and microwave signal skin depth (Penetration depth), as well as attenuation coefficient within soil surfaces. The measurements are depending on the relative dielectric constant that practically carried out by this research. The measurements have been conducted for three common types of soil in Mosul city (ornamental, cropland, and orchard) at $6 \mathrm{GHz}$ (C-band) microwave frequency.

The expected brightness temperatures of these soil samples and the skin depth have been computed. The results show that the observed microwave skin depth is relatively high for ornamental type more than that of cropland and orchard.

Keywords: Moist soil, Penetration depth, Brightness temperature, Complex dielectric constant.

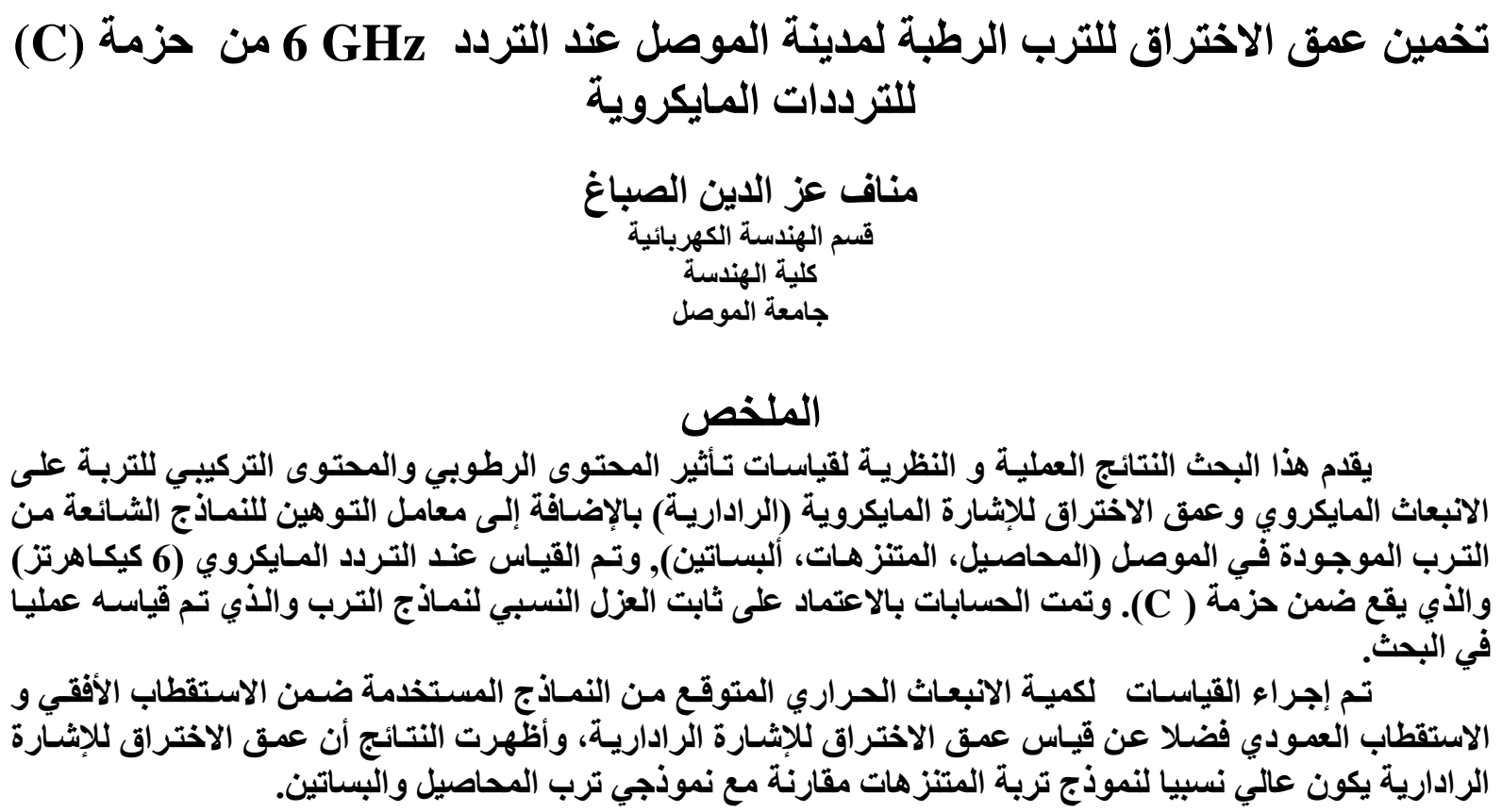




\section{Introduction:}

The soil moisture is difficult to define because it means different things in different disciplines. However soil moisture is the water held in the space between soil particles [1].

Compared to other components of hydrologic cycle, the volume of soil moisture is small, nonetheless, it is of fundamental importance to many hydrological, biological, and into modeling of various ecosystem processes [2]. Despite the importance of soil moisture, widespread and, or continuous measurement of soil moisture is all but nonexistent.

Remote sensing of soil moisture from the vantage point of space is advantageous because of its spatial coverage and temporal continuity [3]. Radiometric microwave remote sensors a board earth orbiting satellites have potential for providing soil moisture information, with the necessary temporal and spatial coverage. The potential of microwave techniques lies in the marked effect of soil moisture on the soil emission and the skin depth (penetration depth) properties at microwave frequencies [4].

The frequency of incident has a direct relationship with the penetration depth in the surface. The $\mathrm{L}$ and $\mathrm{C}$ band widths are most commonly used wavelength for soil moisture estimation [5].

The purpose of this paper is to study the effects of soil moisture content on the radiometric microwave emission properties (reflectivity and brightness temperature) and the skin depth for three types of common existing soils in Mosul city, namely cropland, orchards, and ornamental.

The practical measurements of the real and imaginary parts of complex dielectric constant as a function of soil moisture for the above types of soil have been carried out. The measurements were performed at a microwave frequency of $(6 \mathrm{GHz})$ which is available in the laboratory.

\section{Laboratory measurements and analysis:}

\subsection{Soil samples}

Soil is defined as the external disassembled layer for earth surface, which have difference from deep rocky layers. The soil consist of five main materials; mineral material, water, air, organic material, and biological beings[6].The mineral material that represented the ratio between sand, silt, and clay in one side, and the organic material that reflect amount of carbon in the other side, are co nstant in one location. While each amount of water and air varies in soil for the same location [7].

This study included the collection of three soil samples to represent three types of soil; ornamental, orchards, and cropland. These types of soil have a wide appearance of soil in Mosul city.

\subsection{Relative dielectric constant measurements:}

Water in the soil changes the microwave dielectric constant, which in turn change the emission. Several dielectric mixing models have been developed and evaluated to describe soil-water systems [8].

There are many techniques for dielectric constant measurements, for example the lumped circuit techniques are only suitable for low frequencies and high loss materials, in a cavity perturbation technique, the resonant cavity size may be in term of centimeters at microwave frequencies and the size of the material sample must be much smaller than the cavity size.[9].In this paper the measurement of relative dielectric constant for the three adopted types of soil have been carried out as a function of moisture content

(0- 0.30$)$ by using the shorted-line technique as it available in the laboratory. The principles of this technique are described in detail in reference [10]. 
The measurements were made by packing the moist samples in $(3-\mathrm{cm})$ length waveguide section.

The basic arrangement of the short-line technique used to measure the dielectric properties of soil is shown in Fig. (1)

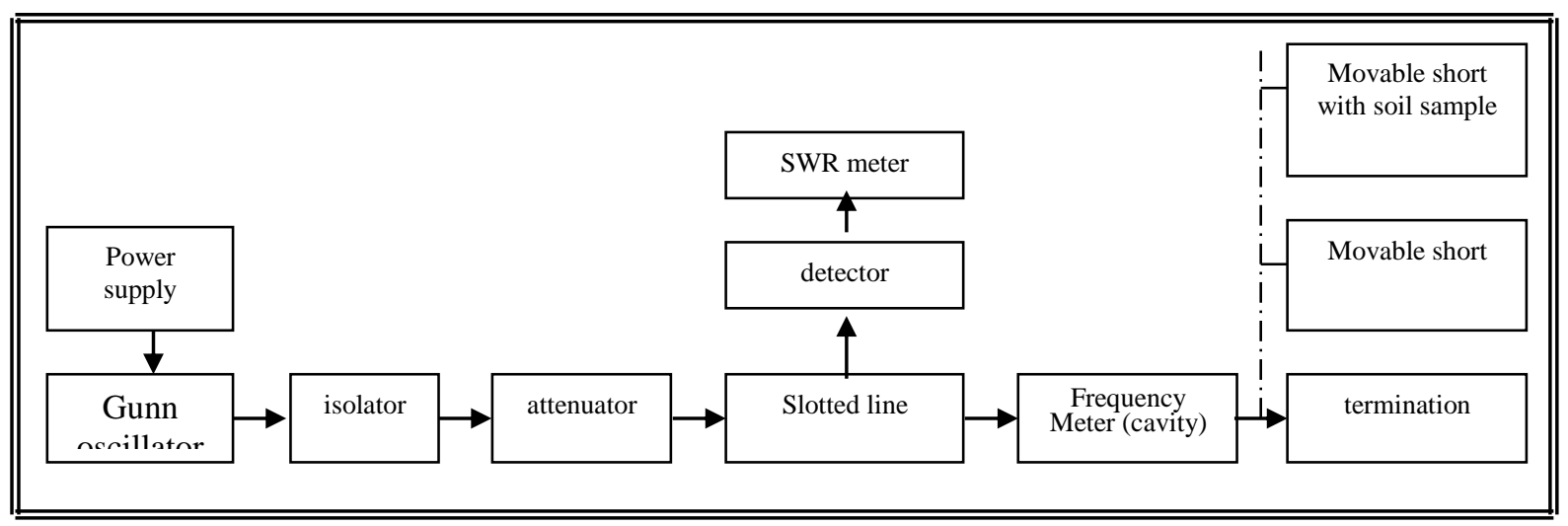

Fig. (1) (a)

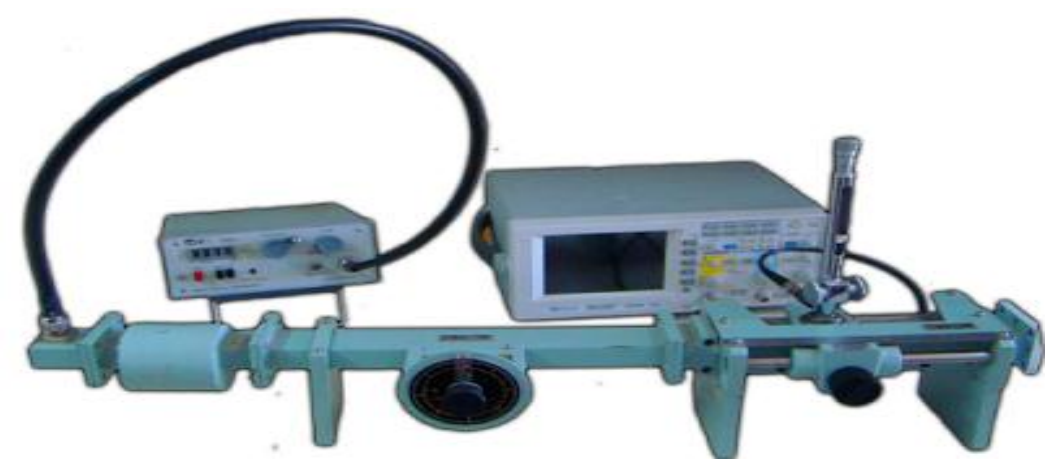

Fig. (1) (b)

Fig. (1) (a): Schematic diagram of the experimental set-up (b)Microwave set-up

In this technique, a slotted line section is used to measure the shift in minimum of a standing wave.

The minima of the standing-wave pattern occur at intervals of one-half wavelength from the short circuit when the soil sample is absent. . By putting the soil sample in the long wave guide section in the front of the short circuit, the minima shift toward the short-circuit as shown in figure (2).

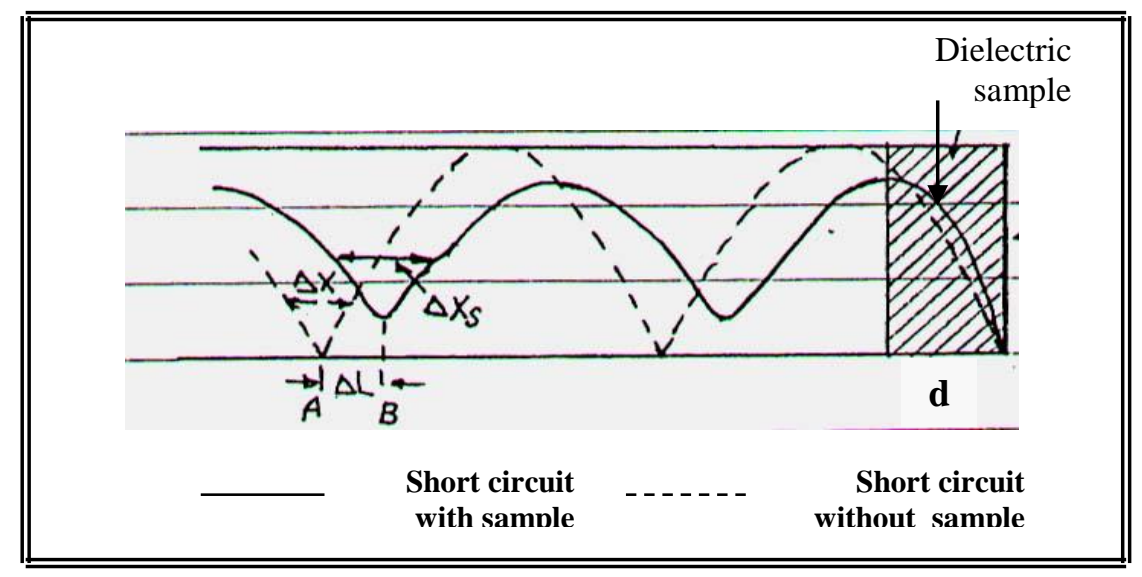

Fig. (2): Standing waves in the waveguide with and without the sample. 
In general, the relative dielectric constant of any material is given by $\varepsilon_{r}=\varepsilon^{\prime}-j \varepsilon^{\prime \prime} \quad$ where $\varepsilon^{\prime}$ the real part, and $\varepsilon^{\prime \prime}=$ the imaginary parts of relative dielectric constant.

The shift in minimum is a measure of the dielectric constant and is given by [10] :

$\varepsilon^{\prime}=\left(\frac{x \lambda}{2 \pi d}\right)^{2}+\left(\frac{\lambda}{\lambda_{c}}\right)^{2}$

Where $\frac{\lambda}{\lambda_{c}}$ is the waveguide proportionality constant which is a function of the waveguide dimensions ( $\lambda_{c}=2 a, a$ being the width of the rectangular waveguide ) at the mode of propagation $\mathrm{TE}_{10}$ and ( $\lambda$ is the operating wavelength ).

$d$ is the length of the soil sample .

$x$ is the multi-valued which can be calculated from the following equation[10];

$\frac{\tan x}{x}=\frac{\lambda_{g}}{2 \pi d} \tan \frac{2 \pi(\Delta L+d)}{\lambda_{g}}$

where,

$\Delta L$ is the shift in the minimum (Fig. (2)).

$\lambda_{g}$ is the waveguide inside the guide.

The signal that is lost in the form of heat in the dielectric causes a decrease in the standing-wave ratio[8]:

$\tan \delta=\frac{\Delta x_{s}-\Delta x}{\varepsilon^{\prime} d}\left(\frac{\lambda}{\lambda_{g}}\right)^{2}$

where,

$\Delta x_{s}, \Delta x$ denote the distances for a fixed voltage standing-wave ratio (VSWR) on either side of the minimum(Fig.(2)).

Then, the loss factor $\left(\varepsilon^{\prime \prime}\right)$ is calculated by;

$\varepsilon^{\prime \prime}=\tan \delta^{*} \varepsilon$

and the relative dielectric constant is given by:

$\varepsilon_{r}=\varepsilon^{\prime}-j \varepsilon^{\prime \prime}$

\section{Microwave Radiative Properties of Soil:}

In passive microwave remote sensing, the data that a radiometer provides is commonly measured and expressed in terms of emissivity.

Emissivity can be predicted from the dielectric constant for a wide variety of conditions. The simplest situation occurs when the dielectric constant of the moisture soil is uniform with depth[8].Under this condition the Fresnel equations can be used to predict emissivity for any incident angle or polarization.

Microwave brightness temperature $(\mathrm{Tb})$ for a smooth surface is related to the relative dielectric constant $\left(\varepsilon_{r}\right)$ through the reflectivity given by[11] :

$T_{b}(\theta, P)=(1-R(\theta, P)) * T$

Where,

$\theta$ is the incident angle

$P$ is the polarization

$R \quad$ is the reflectivity, and

$T \quad$ is the actual soil temperature 
The Fresnel equations that relate $\left(\varepsilon_{r}\right)$ to $(R)$ are given by [12]:

$R(\theta, H)=\left(\frac{\cos \theta-\left(\varepsilon_{r}-\sin ^{2} \theta\right)^{0.5}}{\cos \theta+\left(\varepsilon_{r}-\sin ^{2} \theta\right)^{0.5}}\right)^{2}$

for horizontal polarization, while for vertical polarization is;

$R(\theta, V)=\left(\frac{\varepsilon_{r} \cos \theta-\left(\varepsilon_{r}-\sin ^{2} \theta\right)^{0.5}}{\varepsilon_{r} \cos \theta+\left(\varepsilon_{r}-\sin ^{2} \theta\right)^{0.5}}\right)^{2}$

In this study, equations (6 through 8 ) have been adopted for calculating the emissivity and brightness temperature of the soil samples depending on the measured values of the relative dielectric constant.

\section{Penetration depth model:}

Electromagnetic energy incident normally upon the surface of a homogeneous soil model is partly reflected and partly transmitted through the medium. If the medium is conductive, the transmitted portion will undergo attenuation at a rate defined by the field (electric or magnetic) attenuation $\operatorname{coefficient}(\alpha)$.

Since the attenuation rate is exponential, then the field magnitude at a depth $(\delta=1 / \alpha$ )will reduce to $1 / e(0.37)$ of its surface value and the power will reduce to $1 / e^{2}(0.135)$ of the power at the surface[8].

Hence neglecting contributions from deeper layers represents an omission of less than $2 \%$ of the returned power. The attenuation coefficient, $\alpha$, in nepers $/ \mathrm{m}$ is defined in terms of the operating wavelength (in meter), real and imaginary parts of the complex relative dielectric constant, $\varepsilon^{\prime}$ and $\varepsilon^{\prime \prime}$ respectively as[13]:

$\alpha=\frac{2 \pi}{\lambda} \sqrt{\frac{\varepsilon^{\prime}}{2}\left(\sqrt{1+\left(\frac{\varepsilon^{\prime \prime}}{\varepsilon^{\prime}}\right)^{2}}-1\right)}$

and the Skin depth $(\delta)$ is given by:

$\delta=\frac{1}{\alpha}$

\section{Results and Discussion:}

The theory behind microwave remote sensing of moist soil is based on the large contrast between the dielectric properties of liquid water $(\sim 81)$ and dry soil $(<4)[13],[14]$.

The dielectric properties have been studied and measured in this paper to determine the microwave brightness temperature and the skin depth for common types of soil in Mosul City at a microwave frequency of $6 \mathrm{GHz}$ in the C-band frequency range.

Table (1): Measured values of complex relative dielectric constant as a function of water content

\begin{tabular}{|c|c|c|c|c|c|c|}
\hline \multirow{2}{*}{$\begin{array}{c}\text { Water content, } \\
\mathbf{w}_{\mathbf{c}}\left(\mathbf{c m}^{\mathbf{3}} / \mathbf{c m}^{\mathbf{3}}\right)\end{array}$} & \multicolumn{2}{|c|}{ Cropland } & \multicolumn{2}{c|}{ Ornamental } & \multicolumn{2}{c|}{ Orchard } \\
\cline { 2 - 7 } & $\varepsilon^{\prime}$ & $\varepsilon^{\prime \prime}$ & $\varepsilon^{\prime}$ & $\varepsilon^{\prime \prime}$ & $\varepsilon^{\prime}$ & $\varepsilon^{\prime \prime}$ \\
\hline $0 \%$ & 3.145 & 0.018 & 3.038 & 0.011 & 3.321 & 0.029 \\
$10 \%$ & 3.861 & 0.036 & 3.324 & 0.030 & 4.360 & 0.045 \\
$20 \%$ & 6.772 & 0.053 & 4.982 & 0.035 & 8.872 & 0.062 \\
$30 \%$ & 11.553 & 0.087 & 7.872 & 0.054 & 12.608 & 0.101 \\
\hline
\end{tabular}


As shown in Table (1), the relative dielectric constant $\left(\varepsilon_{r}\right)$ of soil varies with volumetric moisture content.

However, Fig. (3) Indicate clearly that real and imaginary parts of the relative dielectric constant of the three types of soil increase with the increase water content. Also it is found that the real and imaginary parts of the dielectric constant for the soil types of orchard and cropland are higher than that of ornamental (figures (3a) and (3b), respectively). This is probably due to the fact that the specific surface area increases as soil particle size decrease from sand to clay size and is even large for expanding layer lattice clays. The results show that the salinity factor play a minor role as compared to the soil content and texture to influence on the soil dielectric constant.

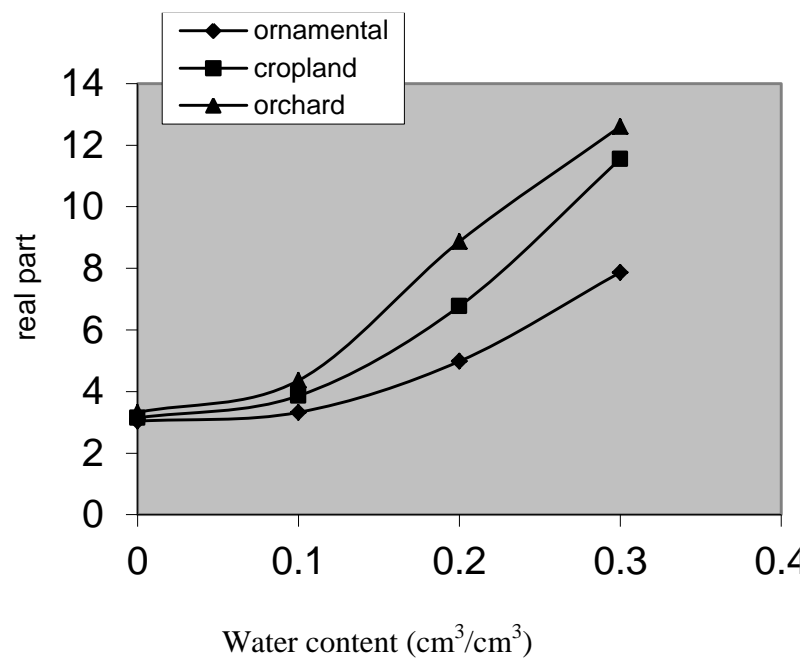

Figure (3a)

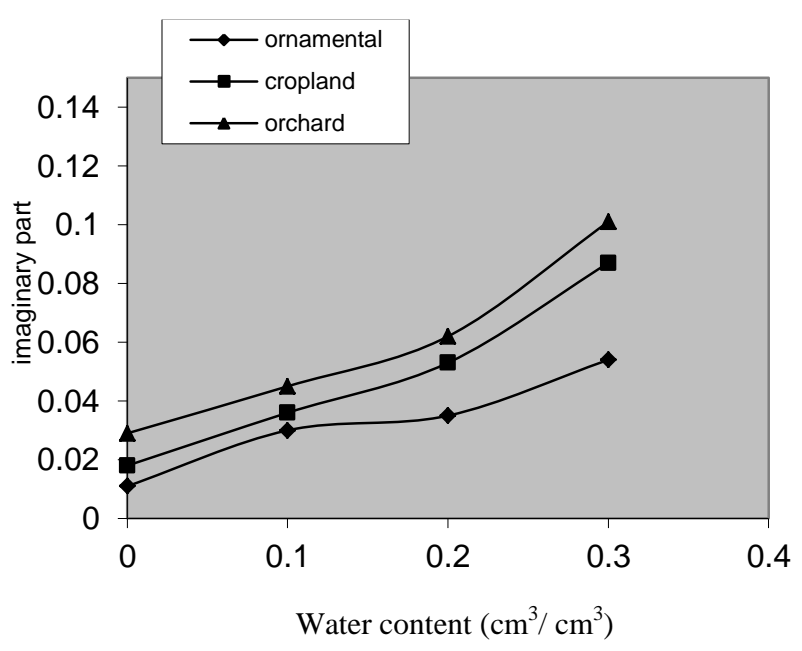

Figure (3b)

Figure (3): Relative dielectric constant of cropland, orchard, and ornamental as a function of water content at $6 \mathrm{GHz}$ :

(a) Real part of relative dielectric constant

(b) Imaginary part of relative dielectric constant

As water in soil changes the dielectric constant, it changes the brightness temperature emission of the soil that measured by radiometer. Fig. (4) shows the brightness temperature variation with moisture content for the samples adopted in this paper at incidence angle $\left(40^{\circ}\right)$ and $\left(60^{\circ}\right)$ as indicated in figure (4a and $4 \mathrm{~b}$, respectively). The calculation was taken for vertical and horizontal polarization as well as physical temperature of soil in order to derive meaningful soil moisture information from the microwave radiometer data.

The data of Fig.(4) demonstrates the inverse relationship that exists between brightness temperature and soil moisture at the two polarizations and that soil ornamental indicates relatively more brightness temperature than cropland and orchard. At the two incident angles, data of the brightness temperature for the three samples are seen to be approximately close to each other, especially at vertical polarization which gives results at $\left(60^{\circ}\right)$ incident angle, this result give a good agreement with the results reported by[15]. 


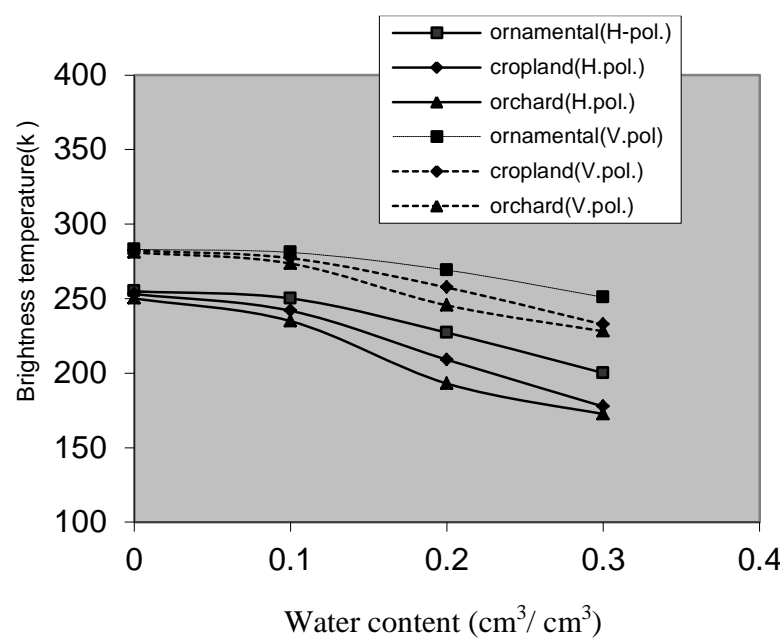

Figure: (4a)

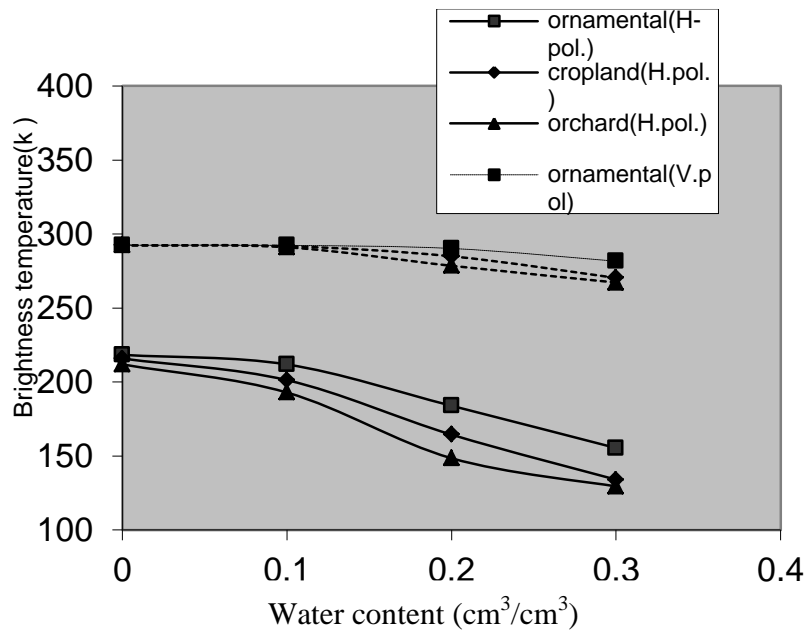

Figure: (4b)

Figure (4): Change of brightness temperature from $\left(20.4^{\circ} \mathrm{C}\right)$ versus soil water content for vertical and horizontal polarization for incident angle of (a) $40^{\circ}$ (b) $60^{\circ}$

Fig. (5) shows increasing of attenuation coefficient with the increasing of soil water content for the three soil samples tested. It is shown that the ornamental exhibited low attenuation coefficient than that of cropland and orchard samples at the given values of water contents, then the ornamental sample gives a relatively high skin depth as compared to other samples studied in this research as shown in Fig. (6). This is due to ability of microwave signal to penetrate in soil depend on the a mount of water in a unit volume of soil as well as the sand content of soil under investigation that characterized by continuity porous. Since the effective depth of penetration of microwave signal increase as increase of sand ratio and decrease of water amount, the observed signals were correlated with the moisture in skin depth as characterized by the attenuation coefficient (reciprocal of skin depth).

Fig.(6) indicates that at

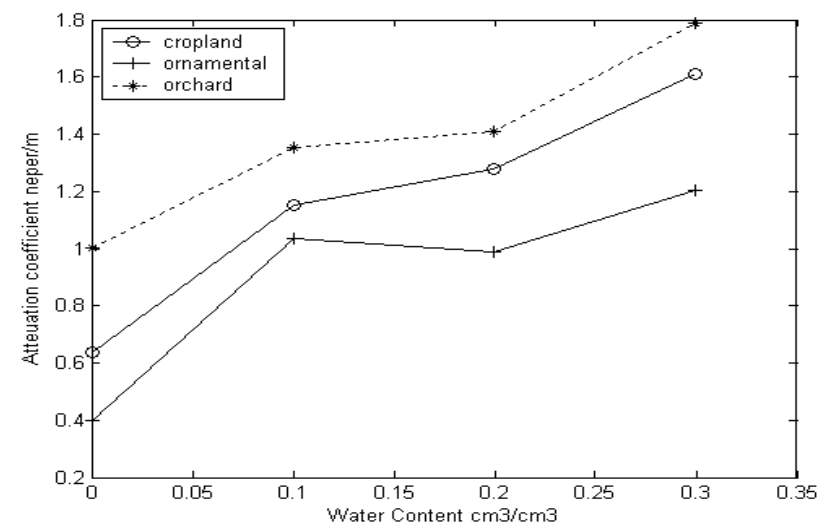

Figure (5): attenuation coefficient variation as a function of water content

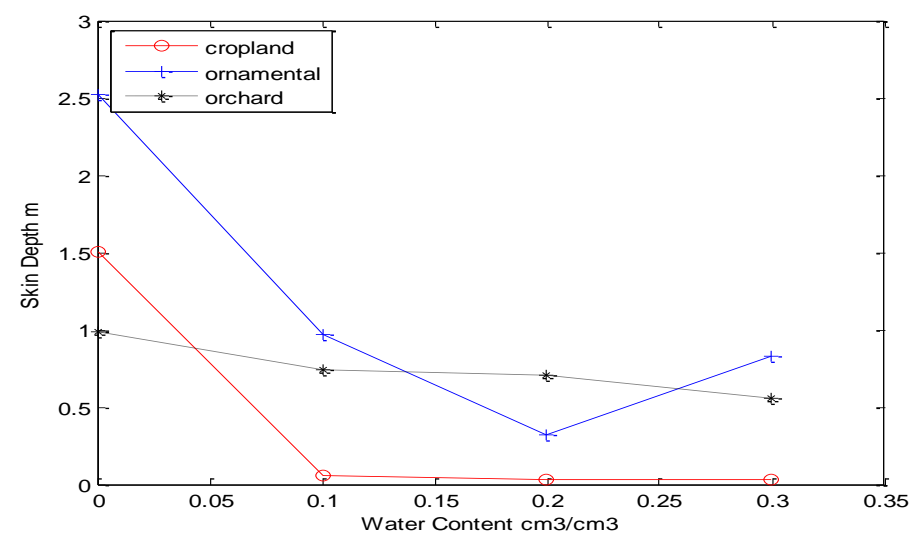

Figure (6): skin depth variation as a function of water content region to distinguish between the three types of Soil. 


\section{Conclusions:}

Measurements have been made of the relative dielectric constant for three types of soil (ornamental, cropland, and orchard) from Mosul city at $6 \mathrm{GHz}$ (C-band). The relative dielectric constant is found to be mainly affected by soil water content .

The predicted skin depth for the ornamental as a function of soil water content $\left(0 \% \sim 30 \% \mathrm{~cm}^{3} / \mathrm{cm}^{3}\right)$ is only of a centimeter order which relatively more than cropland and orchard samples. The results indicate that at moisture content $(10 \%-20 \%)$ my be adequate region to distinguish between the three samples and so we can predict soil type from the skin depth and attenuation coefficient data set.

\section{References:}

1. Leonaro, L. L., 2002. "Mobile field systems for rapid subsurface data acquisition using electromagnetic induction and ground-penetrating radar". M.Sc. Thesis, The University of Tennessee Library, Knoxrille, TN-76P.

2. Eni, G.N., and Peggy, E.O., 1982. "Multifrequency Microwave Radiometer measurements of soil moisture". IEEE Trans. Geoscience and Remote Sensing, Vol. GE-20, No-4, October, PP. 468-475.

3. Farnsworth, R. k., Barreft, E.C., and Dhansu, M.S., 1989." Application of remote sensing to hydrology including ground water". International Hydrological program, Unison, Paris, P. 122.

4. Wang, J., R., Shiue, J., Engman, E., McMurtrey, J., Lawless, P., Scmugge, T., Jackson, T., Gould, W., Fuchs, J., Calhoon, C., Carnahan, T., Hirschman, E., and Glazar, W.," Remote measurements of soil moisture by microwave radiometers at bare test site". NASA Technical Memorandum 80720,21 P. 2005.

5. Lakhankar, T. Krakauer, N. Khanbilvardi, R. , "Application of Microwave Remote Sensing of Soil Moisture for Agricultural Applications", NOAA-Cooperative Remote Sensing Science and technology Center, City University of New York, International Journal of Terraspace Science and Engineering 2 (1) 2009, PP. 81-91.

6. Alexander, M., 1982." Soil microbiology". John Wiley and Sons, INC. USA, P. 573.

7. Deming, D., 2002. "Introduction to hydrogeology". Library of Congress Cataloging-in-Publication Data. McGraw Hill. P. 468.

8. William, L.,C., and Charles, A., L., 2005. "Comparison of two microwave radio brightness models and validation with field measurements". Available at:charles.laymon@msfc.nasa.gov., accessed at: 28/May/2005.

9. Chung B.K, 2007, "Dielectric Constant Measurement For Thin Material At Microwave Frequency ", Progress In Electromagnetics Research, PIER 75, 239252, 2007 , Faculty of Engineering, Multimedia University 63100 Cyberjaya, Malaysia.

10. Younis, H., M., 1983. "Some Effects of Sand and Dust Storms on Iraq National Microwave Links". M. sc. Thesis, Electrical Engineering, University of Mosul.

11. Jackson, T., J., and O'Neill, P., E., 1987. "Salinity effects on the microwave emissivity of soil". IEEE Trans. Geoscience and Remote Sensing, Vol.-25, No.2, March, PP.214-220

12. Zhao, B., Zhao, W., and Du, J., 1983. "Microwave Remote Sensing Of Oil Slick On Water Surface". Scientia Sinica(series A), Vol.xxvi, no.9, pp.978-989

13. Ulaby, F.T., Moore, R. K., and Fung, A. K., 1986. "Microwave remote sensing active and passive". Vol.3, From theory to application, Canada: Dedham MA: Artech House, PP. 2020-2022.

14. Harrington, F. Roger 1991 "Time Harmonic Electromagnetic fields." Wiely-IEEE press

15. Thomas, H, 2003,"Measuring surface soil parameters using passive microwave remote sensing ", HD-No.639, Vrije Universities, Amsterdam.

The work was carried out at the college of Engineering. University of Mosul 